\title{
Magnetic moment measurement in ${ }^{72} \mathrm{Zn}$ using the Transient Field technique and Coulomb excitation in inverse kinematics
}

\author{
A. Illana Sisón ${ }^{1, a}$, A. Jungclaus ${ }^{1}$, R. Orlandi ${ }^{1}$, A. Perea ${ }^{1}$, J.A. Briz' ${ }^{1}$, C. Bauer ${ }^{2}$, R. Gernhäuser ${ }^{3}$, \\ J. Leske ${ }^{2}$, D. Mücher ${ }^{3}$, J. Pakarinen ${ }^{4}$, N. Pietralla ${ }^{2}$, M.M. Rajabali ${ }^{5}$, D. Seiler ${ }^{3}$, and C. Stahl ${ }^{2}$ \\ ${ }^{1}$ Instituto de Estructura de la Materia, CSIC, E-28006 Madrid, Spain \\ ${ }^{2}$ Institut für Kernphysik, Technische Universität Darmstadt, D-64289 Darmstadt, Germany \\ ${ }^{3}$ Physik-Department E12, Technische Universität München, D-85748 Garching, Germany \\ ${ }^{4} \mathrm{CERN}, \mathrm{CH}-1211$ Geneva 23, Switzerland \\ ${ }^{5}$ Instituut voor Kern- en Stralingsfysica, Katholieke Universiteit Leuven, B-3001 Leuven, Belgium
}

\begin{abstract}
The $\mathrm{g}$ factor of the first excited $2^{+}$state of ${ }^{72} \mathrm{Zn}$ has been measured using the Low Velocity Transient Field (LVTF) technique in combination with Coulomb excitation in inverse kinematics. The aim of the experiment was to test the viability of this method when applied to short-lived radioactive ISOL beams, in particular in comparison to the alternative High Velocity Transient Field (HVTF) technique using fragment beams. The result obtained for $g\left(2^{+}\right)$in ${ }^{72} \mathrm{Zn}$ in the present experiment follows the trend observed for the lighter stables $\mathrm{Zn}$ isotopes.
\end{abstract}

\section{Introduction}

The neutron-proton interaction is responsible for changes in the single-particle energies which subsequently modify the sizes of the shell and sub-shell gaps. The neutron-rich region around ${ }^{68} \mathrm{Ni}$ is the object of considerable study and the magic character of this nucleus has been extensively discussed [1]. The $2_{1}^{+}$energies and $\mathrm{B}(\mathrm{E} 2)$ values throughout the chain of $\mathrm{Ni}$ isotopes (see Figure 10 in reference [2]) suggest a sub-shell closure at $\mathrm{N}=40$.

However, the addition of two protons in the $\mathrm{Z}=30 \mathrm{Zn}$ isotopes seems to be already sufficient to destroy this spherical $\mathrm{N}=40$ gap as evidenced by the increasing $\mathrm{B}(\mathrm{E} 2)$ strength when going from ${ }^{68} \mathrm{Zn}$

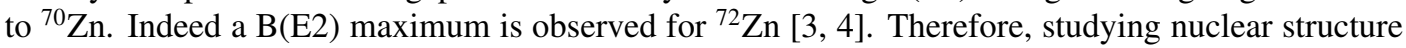
along the $\mathrm{Zn}$ chain provides information about the influence of $\mathrm{N}=40$ in the vicinity of $\mathrm{Z}=28$, i.e. where a few valence particles can be expected to have largest contributions.

Nuclear magnetic moments are very sensitive probes of the contributions of the valence protons and/or neutrons to the wave function of the state of interest, especially in the vicinity of shell closures. The present work is centred on the measurement of the magnetic moment of the first excited $2^{+}$state in ${ }^{72} \mathrm{Zn}$, characterized by a lifetime in the order of ps. In principle three different techniques are available to measure magnetic moments of short-lived excited states: Recoil In Vacuum (RIV), Low Velocity Transient Field (LVTF) and High Velocity Transient Field (HVTF). So far only few experiments have been performed employing these techniques in conjunction with radioactive ions beams [5-10] and

\footnotetext{
a e-mail: andres.illana@csic.es
} 
it is therefore important to study their advantages and disadvantages for different cases. Here we have chosen the LVTF technique in combination with Coulomb excitation in inverse kinematics. This technique has been successfully applied in a large number of stable ion beam experiments in the past [11]. However, due to the inherent use of very thick targets, severe problems due to the accumulation of activity in the target region are expected when applied to short-lived radioactive beams. Therefore so far this technique only has been applied to radioactive beams in a few cases $[5,9,10]$. For the present experiment a new reaction chamber has been constructed in order to minimize as much as possible the deposition of radioactive ions in the field vision of the Ge detectors and thus investigate the feasibility of LVTF experiments for more exotic isotopes. The $2^{+}$g-factor in ${ }^{72} \mathrm{Zn}$ was chosen because it has been measured before using the HVTF method at GANIL [12] allowing for a direct comparison between these two techniques.

\section{Experimental setup}

The experiment was performed at the REX-ISOLDE facility at CERN. A radioactive ${ }^{72} \mathrm{Zn}$ beam, accelerated to an energy of $2.94 \mathrm{MeV} / \mathrm{u}$ with a maximum beam intensity of $9 \cdot 10^{6}$ ions/s, impinged on two different multilayer targets. These targets consisted of a natural carbon layer $\left(1.0 / 0.45 \mathrm{mg} / \mathrm{cm}^{2}\right)$ to Coulomb excite the $\mathrm{Zn}$ ions, a Gd layer $\left(6.7 / 11.8 \mathrm{mg} / \mathrm{cm}^{2}\right)$ magnetized by an external magnetic field in which the excited $\mathrm{Zn}$ ions experience the transient field and a Ta layer $\left(1.0 / 1.0 \mathrm{mg} / \mathrm{cm}^{2}\right)$ which is necessary in the production process of the targets. The targets were cooled by liquid nitrogen to well below the Curie temperature of Gd. Using the target with the thick Gd layer the excited ${ }^{72} \mathrm{Zn}$ ions are stopped in the target before the emission of the $\gamma$-rays of interest as it is usually done in stable beam LVTF experiments. However, such a thick target leads to a significant straggling of the radioactive beam and as a consequence to an activity build up in the target chamber. Therefore, a thinner target was tested as alternative from which the excited ${ }^{72} \mathrm{Zn}$ ions recoil into vacuum. In this case the activation problem is less severe but on the other hand the precession effect is smaller (less interaction time between the magnetic moment of the excited state and the transient field) and the

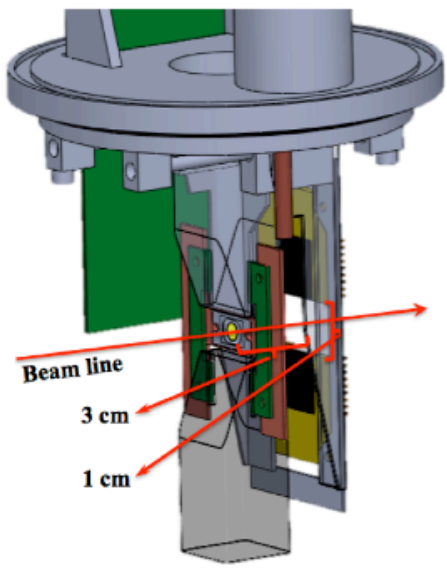

(a)

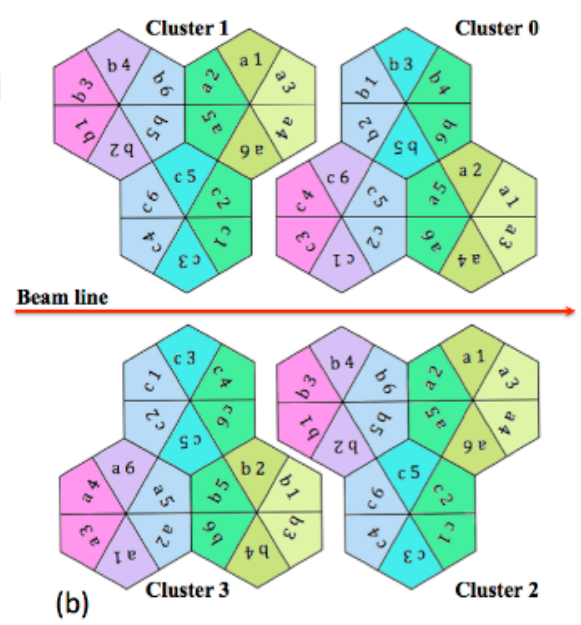

(b)

Figure 1. Sketch of the experimental setup. The position of the DSSSD's with respect to the target (a) and the position of the MINIBALL clusters with respect to the beam (b). 
anisotropy of the angular correlation (and consequently the sensitivity) may be reduced due to RIV effects. It has been the purpose of this experiment to define optimum conditions for this type of experiment.

The different targets were mounted in the new target chamber which also included two square $\left(2 \times 2 \mathrm{~cm}^{2}\right)$ segmented Si detectors for the detection of the scattered carbon ions. They were positioned $3 \mathrm{~cm}$ downstream from the target $1 \mathrm{~cm}$ above and below the beam axis, respectively, covering an angular range from 20 to 45 degrees (see Fig.1a). This geometry selects the reactions with largest spin alignment perpendicular to the beam trajectory [11]. The $\gamma$-rays emitted after the Coulomb excitation of ${ }^{72} \mathrm{Zn}$ were detected by four MINIBALL Cluster detectors. These clusters were positioned at $\pm 60^{\circ}$ and $\pm 120^{\circ}$ with respect to the beam axis in a horizontal plane at a distance of $9.7 \mathrm{~cm}$ from the target (see Fig.1b). The position of the clusters was chosen based on a compromise between the maximum expected effect and the achievable statistics.

\section{Analysis of the experiment}

Fig. 2a) shows the spectrum of $\gamma$-rays observed in prompt coincidence with the particles detected in the $\mathrm{Si}$ detectors above an energy threshold of $2.5 \mathrm{MeV}$. The particle pattern for both detectors is shown in Fig. 2b). The strongest line in this spectrum at $653 \mathrm{keV}$ corresponds to the $2^{+} \rightarrow 0^{+}$transition in ${ }^{72} \mathrm{Zn}$. Also visible is the $847 \mathrm{keV} 4^{+} \rightarrow 2^{+}$transition which feeds the $2^{+}$state. However its intensity is so low (5\% of the intensity of the $653 \mathrm{keV}$ line) that it can be neglected in the determination of $g\left(2^{+}\right)$. In addition lines belonging to known transitions in ${ }^{78} \mathrm{Se}$ and ${ }^{81} \mathrm{Br}$ are observed in Fig. 2a). These nuclei are populated via incomplete fusion in the first and fusion evaporation reactions in the second case as is illustrated in Fig. 2b), which shows the particle energy detected in the different horizontal strips of the Si detectors.

The precession angle $\Delta \Phi$, and hence $g$, can be deduced from the ratio of the photopeak intensities and the slope of the perturbed angular correlation for each angle of detection:

$$
\Delta \Phi=\frac{1}{S(\theta)} \cdot \frac{\rho-1}{\rho+1}
$$
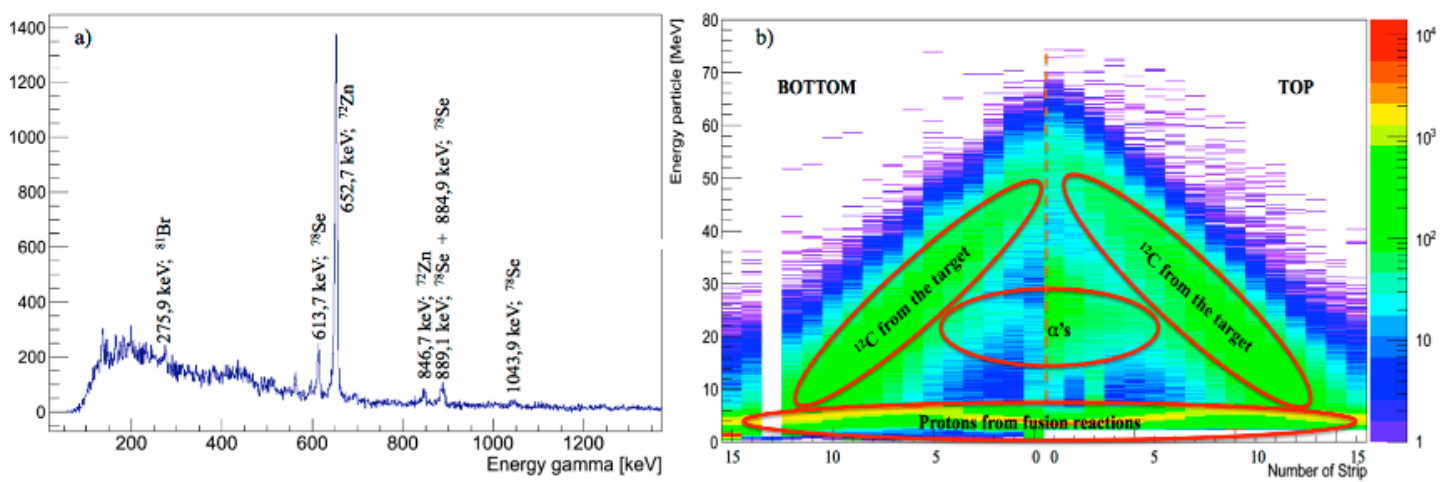

Figure 2. a) Prompt $\gamma$-ray spectrum obtained with the thin target and b) two-dimensional matrix showing the particle energy as a function of the strip number. Strip number 0 corresponds to the minimum angle of about $20^{\circ}$ with respect to the beam. 
here, $\rho$ is the double ratio between the photopeak intensities, $N_{\theta}$, detected in a pair of detectors positioned at angles $\pm \theta$ with respect to the beam close to the horizontal plane for each direction of the vertical magnetic field [13] and $S(\theta)$ is the logarithmic slope of the angular correlation in a plane perpendicular to the magnetic field direction. It can be expressed as $S(\theta)=(W(\theta))^{-1} \cdot(d W(\theta) / d \theta)$ [11].

Finally, the $g$ factor of state of interest can be determined by the experimental precession angle $\Delta \Phi[11]$, as given by (2):

$$
\Delta \Phi=-g \cdot \frac{\mu_{N}}{\hbar} \cdot \int_{t_{\text {in }}}^{t_{\text {out }}} B_{T F}\left(v_{\text {ion }}(t)\right) \cdot e^{-t / \tau} \mathrm{d} t
$$

\section{Conclusions}

As mentioned above two different targets were used in the experiment leading to two completely independent values for $g\left(2^{+}\right)$in ${ }^{72} \mathrm{Zn}$, which are consistent and can be averaged to obtain a final result. However, one can draw some important conclusions by looking at them separately. For the thin target, in which the thickness of the Gd layer is reduced, the integral precession per unit $\mathrm{g}$ factor is 61(9) mrad which has to be compared to a value of 144(16) mrad for the thick target. At the same time the anisotropy of the angular correlation is reduced in the case of the thin target due to a partial deorientation when the ions recoil out of the target into vacuum. These disadvantages are partially compensated by the fact that using the thick target the beam intensity had to be limited in order to keep the activation and consequently the counting rate in the Ge detectors at an acceptable level.

Which approach is better suited in a particular case depends very sensitively on the half-lifes of the isotope of interest and its decay products, the $\gamma$-ray multiplicity within the decay chain and the available beam intensity. The information obtained in the present experiment is very valuable in view of future experiments using the LVTF technique as will be discussed in full detail in a forthcoming publication [14].

This work has been supported by the Spanish Ministerio de Ciencia e Innovación under contracts FPA2009-13377-C02 and FPA2011-29854-C04.

\section{References}

[1] K. Langanke et al., Phys. Rev. C 67, 044314 (2003).

[2] J. Van de Walle et al., Phys. Rev. C 79, 014309 (2009).

[3] M. Niikura et al., Phys. Rev. C 85, 054321 (2012).

[4] C. Louchart et al., Phys. Rev. C 87, 054302 (2013).

[5] G. Kumbartzki et al., Phys. Lett. B 591, 213 (2004).

[6] A.D. Davies et al., Phys. Rev. Lett. 96, 112503 (2006).

[7] A.E. Stuchbery et al., Phys. Rev. C 74, 054307 (2006).

[8] N. Stone et al., Phys. Rev. Lett. 94, 192501 (2005).

[9] N. Benczer-Koller et al., Phys. Lett. B 664, 241 (2008).

[10] G.J. Kumbartzki et al., Phys. Rev. C 86, 034319 (2012).

[11] K.-H. Speidel et al., Prog. Part. Nucl. Phys. 49, 91 (2002).

[12] E. Fiori et al., Phys. Rev C 85, 034334 (2012).

[13] J. Walker et al., Phys. Rev C 84, 014319 (2010).

[14] A. Illana Sisón et al., in preparation 